Article

\title{
Comparison of Different Extraction Methods for the Recovery of Olive Leaves Polyphenols
}

\author{
Ana Dobrinčić ${ }^{D}$, Maja Repajić *(D), Ivona Elez Garofulić, Lucija Tuđen, Verica Dragović-Uzelac \\ and Branka Levaj
}

Faculty of Food Technology and Biotechnology, University of Zagreb, Pierottijeva 6, 10000 Zagreb, Croatia; adobrincic@pbf.hr (A.D.); ielez@pbf.hr (I.E.G.); lucija.tudjen@gmail.com (L.T.); vdragov@pbf.hr (V.D.-U.); blevaj@pbf.hr (B.L.)

* Correspondence: maja.repajic@pbf.unizg.hr

Received: 24 June 2020; Accepted: 17 August 2020; Published: 19 August 2020

\begin{abstract}
In the present study, advanced extraction techniques, microwave (MAE), ultrasound (UAE), and high pressure (HPAE)-assisted extraction, were applied to improve extraction efficiency of olive (Olea europaea L.) leaves polyphenols. The effect of sample mass ( 1.5 and $3 \mathrm{~g})$, MAE-time $(2,8.5$, and $15 \mathrm{~min}$ ) and temperature $\left(45\right.$ and $\left.80^{\circ} \mathrm{C}\right)$, UAE—time $(7,14$, and $21 \mathrm{~min})$ and amplitude (50 and $100 \%$ ) and HPAE - time (1, 5.5, and $10 \mathrm{~min}$ ) and pressure (300 and $500 \mathrm{MPa})$ on the concentration of each analyzed polyphenol compound was examined. Identified polyphenols were oleuropein, hydroxytyrosol, chlorogenic acid, caffeic acid, verbascoside, and rutin. All three advanced extraction techniques yielded higher content of total polyphenols when compared to the conventional heat-reflux extraction (CE) along with a significant reduction of extraction time from 60 (CE) to 2, 21, and $5.5 \mathrm{~min}$ in MAE, UAE, and HPAE, respectively. The most intensive values of tested parameters in each technique were the ones that promoted cell wall disruption, e.g., temperature of $80{ }^{\circ} \mathrm{C}$ in $\mathrm{MAE}, 100 \%$ amplitude in UAE and $500 \mathrm{MPa}$ in HPAE. MAE and UAE were more efficient in total polyphenols' recovery than HPAE.
\end{abstract}

Keywords: olive leaves; polyphenols; oleuropein; microwave-assisted extraction; ultrasound-assisted extraction; high pressure-assisted extraction; heat-reflux extraction; extraction efficiency

\section{Introduction}

Olive (Olea europaea L.) is one of the most important crops in the Mediterranean region primarily used for oil production, where during pruning of the olive tree a significant amount of by-products (leaves and branches) are accumulated. Olive leaves are considered as an inexpensive, renewable, and abundant source of polyphenols [1] and it has been found that olive leaves extract (OLE) has antioxidative, anti-inflammatory, and antimicrobial properties against bacteria and fungi, and also shows anti-viral properties against several different viruses [2]. Phenolic compounds, including phenolic acids, phenolic alcohols, flavonoids and secoiridoids, are the ones responsible for these various positive properties. Several studies have reported the individual phenolic profile of olive leaves showing hydroxytyrosol, rutin, verbascoside, luteolin-7-glucoside, luteolin-4'-glucoside, oleuropein, oleuropein aglycone, and ligstroside aglycone being the most abundant among the large number of diverse phenolic compounds identified [3-8].

OLE polyphenolic content highly depends upon a plant's geographical origin and cultivar, as well as the efficiency of the applied extraction technique. The conventional extraction techniques such as Soxhlet and heat-reflux extraction are usually performed at high temperature, their duration is long and the requirement for sample and solvent, as well as human work, is large, therefore, causing high costs and having a negative impact on the environment and human health [9]. With an intention to solve 
these limitations, some alternative techniques have been developed such as microwave, ultrasound and high hydrostatic pressure-assisted extraction. Each of these techniques has a unique mechanism to improve extraction efficiency. In microwave-assisted extraction (MAE), extraction occurs as the result of changes in the cell structure caused by electromagnetic waves [10], while in ultrasound-assisted extraction (UAE) the enhancement in extraction is mainly attributed to the effect of acoustic cavitation produced in the solvent by the passage of an ultrasound wave [11]. In high-pressure-assisted extraction (HPAE), high pressure disrupts the cell wall and compounds are released from the cytoplasm [12]. Furthermore, the extraction process itself can be affected by the specific extraction parameters such as the sample to solvent ratio, time, temperature, pressure, or ultrasound power. By prolonging the contact of the sample with a solvent or disrupting the cell walls so that more compounds can diffuse to the solvent, more intense extraction conditions (longer extraction time, higher temperature, pressure, or ultrasound power) could possibly improve the extraction yield. However, if applied in excess they could cause the degradation of some polyphenols. All these facts lead to inevitability and necessity for the optimization of the extraction process in order to achieve the maximum effectiveness in isolation of target compounds $[1,4,13]$.

So far HPAE has not been used for the extraction of olive leaves polyphenols, while various studies have dealt with MAE and UAE optimization for olive leaves polyphenols' recovery [1,4,13-15]. However, the optimization of the polyphenols' extraction in the above-mentioned studies was based on the total phenolic content or antioxidant activity of the obtained extracts, without examining the influence of the extraction parameters of these techniques on individual phenolic compounds. Their general conclusion was that in comparison with conventional techniques MAE and UAE are able to provide OLE with similar content of bioactive compounds, but with a remarkable shortening of the extraction time. To the best of our knowledge, there are no studies dealing with the recovery of olive leaves polyphenols involving MAE, UAE, and HPAE simultaneously, as well as their effect on olive leaves individual polyphenols. Therefore, this study aimed to evaluate the effect of various MAE, UAE, and HPAE parameters on the concentration of individual OLE polyphenols as well as to compare these novel extraction techniques mutually and with conventional heat-reflux technique.

\section{Materials and Methods}

\subsection{Chemicals}

Ethanol (96\%) was purchased from Gram-mol d.o.o. (Zagreb, Croatia). HPLC standards of oleuropein $(\mathrm{OL})$, verbascoside $(\mathrm{VB})$, rutin $(\mathrm{RT})$, caffeic acid $(\mathrm{CF})$, and chlorogenic acid (CA) were obtained from Sigma-Aldrich Co. (St. Louis, MO, USA), while hydroxytyrosol (HT) was purchased from Extrasyntheses (Genay, France). All the solvents used for HPLC analysis were HPLC grade.

\subsection{Plant Material}

Olive leaves, cv. Oblica, were collected on Hvar, Croatia. Leaves were air-dried and stored in the dark at ambient temperature. Prior to extraction, leaves were ground with an electric grinder (CM3260, Grunding, Neu-Isenburg, Germany) and the obtained powder was immediately used for the extraction.

\subsection{Extraction Conditions}

The extraction conditions for MAE, UAE, and HPAE were selected based on the previous studies reporting the range of extraction parameters as the most successful for olive polyphenols' extraction $[1,4,7,15,16]$ and, therefore, were taken as the framework for the experimental design applied in this study. For all extraction methods, various sample masses (1.5 and $3 \mathrm{~g})$ were treated with an aqueous solution of ethanol $(50 \%, v / v)$ used as an extraction solvent.

MAE was performed on Start S Microwave Labstation for Synthesis (Milestone, Bergamo, Italy). Extraction parameters were irradiation time $(2,8.5$, and $15 \mathrm{~min})$ and temperature $\left(45\right.$ and $\left.80^{\circ} \mathrm{C}\right)$. 
Microwave power was kept constant at $300 \mathrm{~W}$ until the target temperature was achieved. General extraction parameters were: time to achieve extraction temperature ( $2 \mathrm{~min})$, mixing $(50 \%)$, and ventilation after extraction (1 min).

An ultrasonic processor (UP) $400 \mathrm{~S}$ (Dr. Hielscher GmbH, Teltow, Germany) equipped with ultrasonic probe (surface $3.8 \mathrm{~cm}^{2}$ ) immersed $1 \mathrm{~cm}$ into the solution was used for UAE. The maximal nominal output power of the device was $400 \mathrm{~W}$ and the ultrasonic frequency was $24 \mathrm{kHz}$. Extraction time was 7, 14, and $21 \mathrm{~min}$ with an amplitude of 50 and $100 \%$. The temperature was measured with an infrared thermometer and it did not exceed $60{ }^{\circ} \mathrm{C}$.

HPAE was conducted in the FPG7100.100 system (Stansted Fluid Power Ltd., Harlow, UK), where the sample-solvent mixture was vacuum packaged and extracted at different pressures (300 and $500 \mathrm{MPa}$ ) and extraction times (1, 5.5, and $10 \mathrm{~min}$ ) with glycol solution used as a pressure-transmitting media.

The temperature was varied as an extraction parameter only in the MAE technique since MAE offers the possibility of its full control during the process. In UAE, it can be controlled only partially in a temperature range by external cooling during the extraction process (it was measured by an infrared thermometer and kept bellow $60^{\circ} \mathrm{C}$ ), while HPAE was conducted at ambient temperature, where the increase of temperature as a function of pressure did not promote the degradation of analyzed compounds [17]. Therefore, in the UAE and HPAE the temperature was not taken as a variable.

In order to compare the efficiency of extraction methods listed above with a conventional extraction technique, heat-reflux extraction (CE) was also conducted under previously defined optimal conditions (data not shown): $3 \mathrm{~g}$ of the sample was placed into the flask containing $40 \mathrm{~mL}$ of the extraction solvent, extracted for $30 \mathrm{~min}$, and decanted. The extraction of residue was then repeated under the same conditions and extracts from each cycle were combined.

After each extraction, obtained extracts were made up to $100 \mathrm{~mL}$ with the extraction solvent, centrifugated at $5000 \mathrm{rpm} / 10 \mathrm{~min}$ and the resulting supernatants were stored at $-18{ }^{\circ} \mathrm{C}$ until further analysis. Each treatment was done in two replicates $(n=2)$.

\subsection{HPLC Analysis}

An HPLC system (Agilent Technologies HPLC 1200 Series, Santa Clara, CA, USA) equipped with a binary pump, autosampler, UV/Vis-Photo Diode Array Detector (DAD) and a Luna $5 \mu \mathrm{m}$ C18 column ( $250 \mathrm{~mm} \times 4.6 \mathrm{~mm}, 100 \AA$ A (Phenomenex, Torrance, CA, USA) was used for the separation, identification, and quantification of polyphenols according to the method described by Richard et al. (2011) [18] with some modifications. Mobile phases were $0.1 \%$ formic acid in water ( $v / v$; solvent A) and $0.1 \%$ formic acid in methanol $(v / v$; solvent $\mathrm{B}$ ) and the following gradient was used: $0-3 \mathrm{~min}, 10 \% \mathrm{~B}$; 3-30 min, 50\% B; 30-40 min, 60\% B; 40-45 min, 60\% B; $45-50 \mathrm{~min}, 100 \%$ B; 50-60 min, $10 \%$ B. Operating conditions were: constant flow rate $1 \mathrm{~mL} \mathrm{~min}^{-1}$, column temperature $30^{\circ} \mathrm{C}$, injection volume $20 \mu \mathrm{L}$ and UV/Vis-Photo Diode Array detection at $280 \mathrm{~nm}$. Prior to automatic injection into the HPLC system, standards and samples were filtered using $0.45 \mu \mathrm{m}$ nylon membrane.

Identification of polyphenols was carried out by comparing retention times and spectral data of the separated peaks with those of authentic standards. The quantification of polyphenols was performed by the external standard method. All standards were prepared as stock solutions in methanol. Stock solution was diluted to yield five increasing concentrations and five-point calibration curves were used to calculate the amount of each compound. All measurements were performed in duplicate $(n=4)$ and results were expressed as mean value $\pm \mathrm{SD}$ of $\mathrm{mg} \mathrm{g}^{-1}$.

\subsection{Statistical Analysis}

Statistical analysis was carried out using Statistica ver. 8.0 software (Statsoft Inc., Tulsa, OK, USA). Full factorial randomized experimental design was applied, and descriptive statistics were employed for the basic data evaluation. All continuous variables were analyzed by multivariate analysis of variance (MANOVA) and marginal mean values were compared with Tukey's HSD test. Principal 
component analysis (PCA) was conducted in order to examine possible grouping of the samples. All tests were evaluated at the significance level $p \leq 0.05$.

\section{Results and Discussion}

In the present study, different conditions of MAE, UAE, and HPAE were applied for the polyphenols recovery from olive leaves. Extracts were analyzed by HPLC and identified polyphenols, as well as their concentrations, are shown in Table 1. Results present the measurement values of individual phenols in extracts obtained by conducted extraction techniques in which specific extraction parameters were varied. HPLC analysis showed that composition of identified polyphenols remained unchanged for all experimental trials, containing six polyphenols listed according to their retention time: HT, CA, $\mathrm{CF}, \mathrm{VB}, \mathrm{RT}$, and OL (Figures 1 and 2).
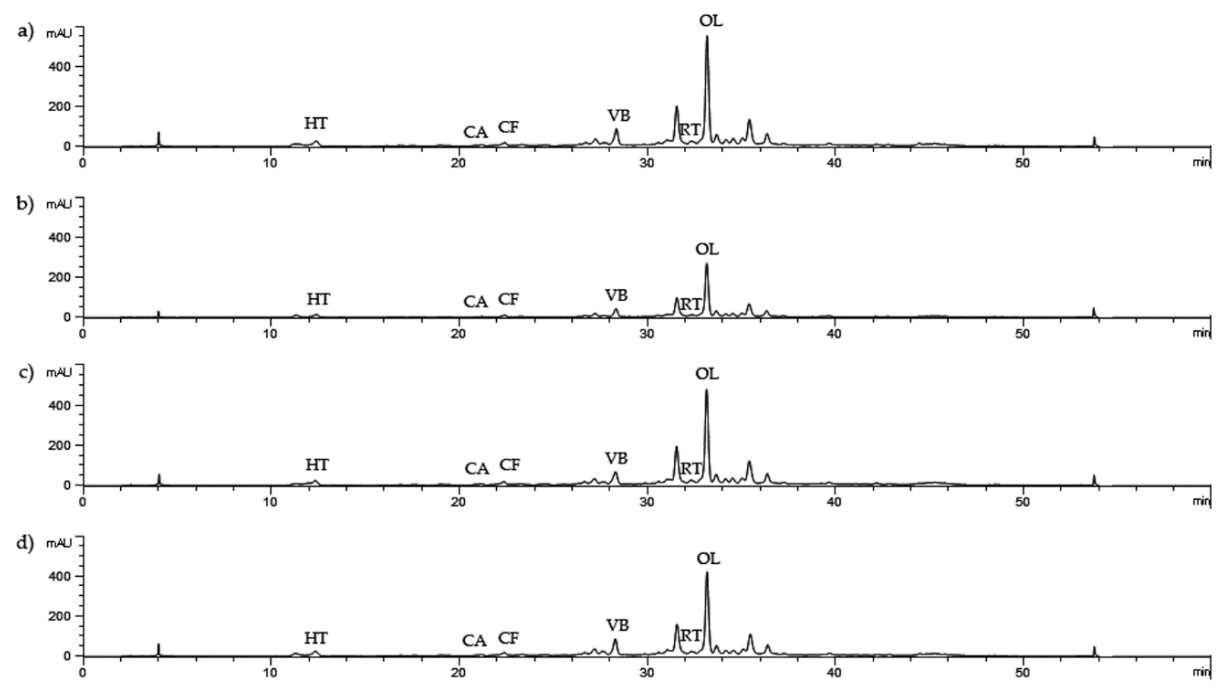

Figure 1. HPLC chromatograms at $280 \mathrm{~nm}$ of olive leaves extracts obtained by (a) microwave-assisted extraction ( $80^{\circ} \mathrm{C}, 2 \mathrm{~min}, 3 \mathrm{~g}$ sample); (b) ultrasound-assisted extraction (50\%, $21 \mathrm{~min}, 1.5 \mathrm{~g}$ sample); (c) high pressure-assisted extraction (300 MPa, $10 \mathrm{~min}, 3 \mathrm{~g}$ sample); (d) conventional heat-reflux extraction $(2 \times 30 \mathrm{~min}, 3 \mathrm{~g}$ sample) $(\mathrm{HT}=$ hydroxytyrosol, $\mathrm{CA}=$ chlorogenic acid, $\mathrm{CF}=$ caffeic acid, $\mathrm{VB}=$ verbascoside, $\mathrm{RT}=$ rutin, $\mathrm{OL}=$ oleuropein).<smiles>OCCc1ccc(O)c(O)c1</smiles>

HYDROXYTYROSOL

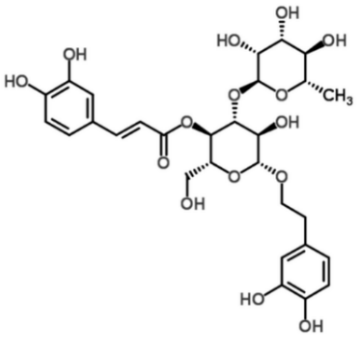

VERBASCOSIDE

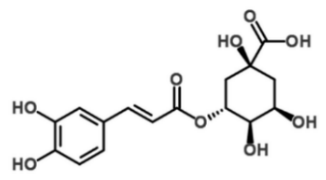

CHLOROGENIC ACID

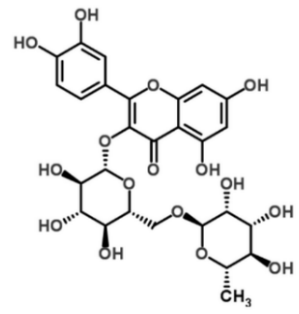

RUTIN<smiles>O=C(O)/C=C/c1ccc(O)c(O)c1</smiles>

CAFFEIC ACID

Figure 2. Chemical structure of polyphenols identified in olive leaves extracts. 
Table 1. Olive leaves individual phenols $\left(\mathrm{mg} \mathrm{g}^{-1}\right)$ extracted by various extraction techniques.

\begin{tabular}{|c|c|c|c|c|c|c|c|c|c|c|}
\hline $\begin{array}{l}\text { Extraction } \\
\text { Technique }\end{array}$ & & Extraction Parame & & OL & HT & CA & $\mathrm{CF}$ & VB & RT & Total Sum \\
\hline \multirow{13}{*}{ MAE } & Time (min) & Temperature $\left({ }^{\circ} \mathrm{C}\right)$ & Mass (g) & & & & & & & \\
\hline & 2 & 45 & 1.5 & $74.11 \pm 0.00$ & $1.10 \pm 0.42$ & $0.44 \pm 0.00$ & $0.26 \pm 0.00$ & $0.50 \pm 0.00$ & $2.57 \pm 0.00$ & $78.98 \pm 0.42$ \\
\hline & 2 & 45 & 3.0 & $69.51 \pm 0.43$ & $0.83 \pm 0.00$ & $0.45 \pm 0.01$ & $0.28 \pm 0.00$ & $0.35 \pm 0.01$ & $2.23 \pm 0.01$ & $73.65 \pm 0.43$ \\
\hline & 2 & 80 & 1.5 & $83.41 \pm 0.06$ & $0.86 \pm 0.00$ & $0.42 \pm 0.00$ & $0.24 \pm 0.01$ & $0.55 \pm 0.01$ & $2.70 \pm 0.02$ & $88.19 \pm 0.09$ \\
\hline & 2 & 80 & 3.0 & $87.75 \pm 0.11$ & $0.95 \pm 0.03$ & $0.46 \pm 0.01$ & $0.32 \pm 0.01$ & $0.41 \pm 0.00$ & $2.30 \pm 0.01$ & $92.18 \pm 0.11$ \\
\hline & 8.5 & 45 & 1.5 & $52.14 \pm 0.04$ & $0.61 \pm 0.01$ & $0.44 \pm 0.00$ & $0.20 \pm 0.00$ & $0.31 \pm 0.00$ & $8.68 \pm 0.06$ & $62.38 \pm 0.02$ \\
\hline & 8.5 & 45 & 3.0 & $65.93 \pm 0.10$ & $0.80 \pm 0.01$ & $0.41 \pm 0.00$ & $0.26 \pm 0.01$ & $0.33 \pm 0.00$ & $2.15 \pm 0.07$ & $69.89 \pm 0.20$ \\
\hline & 8.5 & 80 & 1.5 & $84.82 \pm 0.21$ & $0.94 \pm 0.00$ & $0.45 \pm 0.00$ & $0.29 \pm 0.01$ & $0.55 \pm 0.00$ & $2.43 \pm 0.01$ & $89.49 \pm 0.24$ \\
\hline & 8.5 & 80 & 3.0 & $82.44 \pm 0.57$ & $0.93 \pm 0.03$ & $0.41 \pm 0.02$ & $0.29 \pm 0.00$ & $0.40 \pm 0.00$ & $2.14 \pm 0.27$ & $86.61 \pm 0.89$ \\
\hline & 15 & 45 & 1.5 & $64.03 \pm 0.05$ & $0.82 \pm 0.01$ & $0.44 \pm 0.01$ & $0.24 \pm 0.01$ & $0.47 \pm 0.00$ & $2.76 \pm 0.01$ & $68.75 \pm 0.05$ \\
\hline & 15 & 45 & 3.0 & $70.88 \pm 0.05$ & $0.87 \pm 0.01$ & $0.48 \pm 0.03$ & $0.33 \pm 0.04$ & $0.34 \pm 0.00$ & $2.46 \pm 0.00$ & $75.36 \pm 0.12$ \\
\hline & 15 & 80 & 1.5 & $80.66 \pm 0.25$ & $0.92 \pm 0.00$ & $0.43 \pm 0.03$ & $0.26 \pm 0.01$ & $0.54 \pm 0.00$ & $2.42 \pm 0.03$ & $85.23 \pm 0.25$ \\
\hline & 15 & 80 & 3.0 & $82.01 \pm 0.18$ & $0.82 \pm 0.17$ & $0.44 \pm 0.00$ & $0.30 \pm 0.01$ & $0.42 \pm 0.04$ & $2.42 \pm 0.00$ & $86.41 \pm 0.04$ \\
\hline \multirow{13}{*}{ UAE } & Time (min) & Amplitude (\%) & Mass (g) & & & & & & & \\
\hline & 7 & 50 & 1.5 & $79.28 \pm 0.26$ & $3.10 \pm 1.11$ & $0.46 \pm 0.00$ & $0.33 \pm 0.00$ & $0.50 \pm 0.00$ & $2.54 \pm 0.01$ & $86.20 \pm 0.86$ \\
\hline & 7 & 50 & 3.0 & $76.42 \pm 0.05$ & $2.11 \pm 0.00$ & $0.53 \pm 0.04$ & $0.55 \pm 0.04$ & $0.39 \pm 0.01$ & $2.09 \pm 0.04$ & $82.07 \pm 0.06$ \\
\hline & 7 & 100 & 1.5 & $67.30 \pm 0.28$ & $1.96 \pm 0.21$ & $0.37 \pm 0.01$ & $0.27 \pm 0.01$ & $0.49 \pm 0.01$ & $2.17 \pm 0.00$ & $72.54 \pm 0.08$ \\
\hline & 7 & 100 & 3.0 & $84.62 \pm 0.61$ & $1.69 \pm 0.04$ & $0.59 \pm 0.09$ & $0.51 \pm 0.16$ & $0.42 \pm 0.00$ & $2.06 \pm 0.04$ & $89.88 \pm 0.86$ \\
\hline & 14 & 50 & 1.5 & $60.33 \pm 0.52$ & $1.83 \pm 0.06$ & $0.35 \pm 0.01$ & $0.24 \pm 0.01$ & $0.47 \pm 0.00$ & $2.04 \pm 0.00$ & $65.24 \pm 0.58$ \\
\hline & 14 & 50 & 3.0 & $56.84 \pm 0.08$ & $1.44 \pm 0.01$ & $0.39 \pm 0.01$ & $0.25 \pm 0.01$ & $0.37 \pm 0.01$ & $1.81 \pm 0.00$ & $61.09 \pm 0.04$ \\
\hline & 14 & 100 & 1.5 & $66.01 \pm 0.66$ & $2.21 \pm 0.02$ & $0.42 \pm 0.00$ & $0.29 \pm 0.01$ & $0.52 \pm 0.00$ & $2.37 \pm 0.01$ & $71.81 \pm 0.63$ \\
\hline & 14 & 100 & 3.0 & $81.38 \pm 0.23$ & $2.59 \pm 0.02$ & $0.53 \pm 0.00$ & $0.55 \pm 0.00$ & $0.44 \pm 0.01$ & $2.22 \pm 0.02$ & $87.69 \pm 0.28$ \\
\hline & 21 & 50 & 1.5 & $85.90 \pm 0.04$ & $2.61 \pm 0.01$ & $0.50 \pm 0.01$ & $0.39 \pm 0.01$ & $0.56 \pm 0.01$ & $2.54 \pm 0.01$ & $92.49 \pm 0.04$ \\
\hline & 21 & 50 & 3.0 & $74.35 \pm 0.77$ & $2.32 \pm 0.04$ & $0.48 \pm 0.00$ & $0.37 \pm 0.01$ & $0.39 \pm 0.00$ & $2.00 \pm 0.00$ & $79.90 \pm 0.82$ \\
\hline & 21 & 100 & 1.5 & $77.91 \pm 0.23$ & $2.52 \pm 0.02$ & $0.48 \pm 0.01$ & $0.37 \pm 0.01$ & $0.54 \pm 0.01$ & $2.43 \pm 0.03$ & $84.23 \pm 0.23$ \\
\hline & 21 & 100 & 3.0 & $80.91 \pm 0.92$ & $1.95 \pm 0.00$ & $0.54 \pm 0.08$ & $0.63 \pm 0.00$ & $0.42 \pm 0.00$ & $2.11 \pm 0.02$ & $86.55 \pm 1.02$ \\
\hline \multirow{13}{*}{ HPAE } & Time (min) & Pressure (MPa) & Mass (g) & & & & & & & \\
\hline & 1 & 300 & 1.5 & $69.76 \pm 0.42$ & $1.58 \pm 1.03$ & $0.48 \pm 0.01$ & $0.27 \pm 0.00$ & $0.47 \pm 0.00$ & $2.80 \pm 0.06$ & $75.35 \pm 0.55$ \\
\hline & 1 & 300 & 3.0 & $67.76 \pm 0.49$ & $0.82 \pm 0.00$ & $0.39 \pm 0.01$ & $0.22 \pm 0.01$ & $0.50 \pm 0.00$ & $2.51 \pm 0.06$ & $72.19 \pm 0.44$ \\
\hline & 1 & 500 & 1.5 & $72.19 \pm 0.18$ & $0.85 \pm 0.00$ & $0.43 \pm 0.01$ & $0.25 \pm 0.01$ & $0.51 \pm 0.00$ & $2.61 \pm 0.05$ & $76.82 \pm 0.14$ \\
\hline & 1 & 500 & 3.0 & $72.26 \pm 0.33$ & $0.85 \pm 0.01$ & $0.42 \pm 0.01$ & $0.25 \pm 0.01$ & $0.52 \pm 0.00$ & $2.51 \pm 0.01$ & $76.80 \pm 0.32$ \\
\hline & 5.5 & 300 & 1.5 & $73.00 \pm 0.83$ & $0.85 \pm 0.00$ & $0.45 \pm 0.00$ & $0.25 \pm 0.04$ & $0.50 \pm 0.00$ & $2.60 \pm 0.15$ & $77.64 \pm 1.02$ \\
\hline & 5.5 & 300 & 3.0 & $72.74 \pm 0.25$ & $0.86 \pm 0.01$ & $0.42 \pm 0.03$ & $0.25 \pm 0.01$ & $0.52 \pm 0.00$ & $2.54 \pm 0.02$ & $77.32 \pm 0.30$ \\
\hline & 5.5 & 500 & 1.5 & $66.64 \pm 1.20$ & $0.79 \pm 0.20$ & $0.33 \pm 0.16$ & $0.35 \pm 0.07$ & $0.35 \pm 0.00$ & $2.14 \pm 0.03$ & $70.60 \pm 1.51$ \\
\hline & 5.5 & 500 & 3.0 & $71.47 \pm 0.21$ & $0.85 \pm 0.23$ & $0.22 \pm 0.04$ & $0.32 \pm 0.01$ & $0.38 \pm 0.00$ & $2.27 \pm 0.01$ & $75.50 \pm 0.45$ \\
\hline & 10 & 300 & 1.5 & $66.29 \pm 0.49$ & $0.95 \pm 0.01$ & $0.43 \pm 0.01$ & $0.30 \pm 0.01$ & $0.36 \pm 0.00$ & $2.26 \pm 0.01$ & $70.58 \pm 0.50$ \\
\hline & 10 & 300 & 3.0 & $74.56 \pm 1.98$ & $1.04 \pm 0.00$ & $0.34 \pm 0.13$ & $0.30 \pm 0.00$ & $0.39 \pm 0.01$ & $2.25 \pm 0.05$ & $78.87 \pm 2.05$ \\
\hline & 10 & 500 & 1.5 & $60.48 \pm 0.95$ & $0.77 \pm 0.19$ & $0.39 \pm 0.00$ & $0.26 \pm 0.00$ & $0.33 \pm 0.01$ & $2.04 \pm 0.03$ & $64.26 \pm 0.73$ \\
\hline & 10 & 500 & 3.0 & $63.63 \pm 0.18$ & $0.65 \pm 0.01$ & $0.38 \pm 0.01$ & $0.26 \pm 0.00$ & $0.35 \pm 0.00$ & $1.93 \pm 0.00$ & $67.19 \pm 0.20$ \\
\hline
\end{tabular}

$\mathrm{MAE}=$ microwave-assisted extraction, $\mathrm{UAE}=$ ultrasound-assisted extraction, $\mathrm{HPAE}=$ high pressure-assisted extraction, $\mathrm{OL}=$ oleuropein, $\mathrm{HT}=\mathrm{hydroxytyrosol}, \mathrm{CA}=\mathrm{chlorogenic}$ acid $\mathrm{CF}=$ caffeic acid, $\mathrm{VB}=$ verbascoside, $\mathrm{RT}=$ rutin. Results are expressed as mean $\pm \mathrm{SD}$. 
The total sum of extracted polyphenols ranged from 61.09 to $92.49 \mathrm{mg} \mathrm{g}^{-1}$ (Table 1), which is higher than $52.10 \mathrm{mg} \mathrm{GAE} \mathrm{g}^{-1}$ reported by Talhaoui et al. (2014) [3] but within the range of values reported by Ahmad-Qasem et al. (2013) [13] (66 $\left.\mathrm{mg} \mathrm{g}^{-1}\right)$, Hannachi et al. (2019) [14] (81.96 $\left.\mathrm{mg} \mathrm{GAE} \mathrm{g}^{-1}\right)$, and Rafiee et al. (2011) [15] (69.03 $\mathrm{mg} \mathrm{TAE} \mathrm{g}^{-1}$ ). OL was the most abundant polyphenol, composing between $83.59 \%$ and $95.20 \%$ of all identified polyphenols, followed by RT $(2.29-13.92 \%)$ and HT $(0.95-3.58 \%)$. Present polyphenols, especially OL, which is typical for the Oleaceae family [19], have major biological and health properties [20-22]. The ratio between obtained compounds was also not influenced by the extraction method. Ahmad-Qasem et al. (2013) [13], Mkaouar et al. (2016) [5], Bouaziz and Sayadi (2005) [7], Mitsopoulos et al. (2011) [8], and Dekanski et al. (2009) [23] also confirmed that OL was the major compound in OLE. Other polyphenols identified in this research were also previously identified in OLE by other authors: VB [5,13], HT [5,8], RT [7,8], CA [8], and CF [23].

Based on the results from Table 1, statistical analysis was conducted in order to examine the influence of tested extraction parameters on yield of individual polyphenols, as well as their sum in order to provide information on at which of the tested conditions the highest yields of analyzed compounds were obtained, therefore, allowing an insight into each of the extraction techniques' efficiency. Statistical data are provided in Table 2.

\subsection{Microwave-Assisted Extraction (MAE)}

In order to establish the optimal MAE conditions for OLE polyphenols' extraction, time $(2,8.5$, and $15 \mathrm{~min})$, temperature $\left(45\right.$ and $\left.80^{\circ} \mathrm{C}\right)$ and sample mass $(1.5$ and $3 \mathrm{~g})$ were varied and their influence on the concentration of individual OLE polyphenols is shown in Table 2. Time had a significant influence $(p \leq 0.05)$ on all polyphenols except HT. Extraction of only 2 min resulted in the highest OL concentration and sum of all identified polyphenols. The lowest yields of OL, VB, CF, and CA were obtained after $8.5 \mathrm{~min}$, except for RT which yielded the highest concentration at that extraction time (Table 2). Similarly to our results, Hannachi et al. (2019) [14] reported an optimal time of $1.81 \mathrm{~min}$ for the MAE of polyphenols from olive leaves, while Taamalli et al. (2012) [4], Japón-Luján et al. (2006) [24] and Rafiee et al. (2011) [15] reported a longer time of 6, 8, and $15 \mathrm{~min}$, respectively. Differences in reported results could be attributed to the different extraction conditions used for the extraction $[4,14,15,24]$, especially when MAE duration strongly depends on the applied temperature and/or microwave power. Accordingly, there are two general approaches established regarding the MAE conditions: the first is to apply short, intensive treatment and the second is to apply a prolonged, but moderate one [25].

Concentrations of all identified polyphenols, except HT and CA, were also affected by the temperature. Murakami et al. (2004) [26] also confirmed that temperature does not affect CA concentration since it was stable during heating at $180^{\circ} \mathrm{C}$ for $180 \mathrm{~min} . \mathrm{OL}, \mathrm{VB}$, and $\mathrm{CF}$ concentrations were significantly higher at $80^{\circ} \mathrm{C}$, while RT concentration was higher at $45^{\circ} \mathrm{C}$. Differences in optimal temperature could be explained by the fact that different polyphenols show different sensitivity to the heat treatment depending on their structures [27]. In general, flavonoids (e.g., RT) are more sensitive to thermal degradation than phenolic acids [28]. The elevated temperature usually improves the extraction yield and it shortens the extraction time, but at the same time, intense temperature or prolonged exposure to high temperature can cause a degradation of thermally sensitive compounds and therefore could result in a poor extraction yield [10]. This finding was confirmed by Taamalli et al. (2012) [4] who reported that the extraction efficiency of most of the OLE polyphenols, including OL and RT, increased with the temperature elevation up to $80^{\circ} \mathrm{C}$, while with further temperature increase the extraction efficiency decreased. 
Table 2. Influence of extraction parameters on olive leaves individual phenols $\left(\mathrm{mg} \mathrm{g}^{-1}\right)$.

\begin{tabular}{|c|c|c|c|c|c|c|c|c|}
\hline $\begin{array}{l}\text { Extraction } \\
\text { Technique }\end{array}$ & Source of Variation & OL & HT & CA & CF & VB & RT & Total Sum \\
\hline \multirow{10}{*}{ MAE } & Time (min) & $p<0.01 *$ & $p=0.26 \mathrm{~ns}$ & $p=0.05^{*}$ & $p=0.03 *$ & $p<0.01 *$ & $p<0.01 *$ & $p<0.01 *$ \\
\hline & 2 & $78.69 \pm 0.08 \mathrm{c}$ & $0.93 \pm 0.05 \mathrm{a}$ & $0.44 \pm 0.00 \mathrm{ab}$ & $0.27 \pm 0.00 \mathrm{ab}$ & $0.45 \pm 0.00 \mathrm{~b}$ & $2.45 \pm 0.03 \mathrm{a}$ & $83.25 \pm 0.12 c$ \\
\hline & 8.5 & $71.33 \pm 0.08 \mathrm{a}$ & $0.82 \pm 0.05 \mathrm{a}$ & $0.43 \pm 0.00 \mathrm{a}$ & $0.26 \pm 0.00 \mathrm{a}$ & $0.40 \pm 0.00 \mathrm{a}$ & $3.85 \pm 0.03 \mathrm{~b}$ & $77.09 \pm 0.12 \mathrm{a}$ \\
\hline & 15 & $74.40 \pm 0.08 \mathrm{~b}$ & $0.86 \pm 0.05 \mathrm{a}$ & $0.45 \pm 0.00 \mathrm{c}$ & $0.28 \pm 0.00 \mathrm{c}$ & $0.44 \pm 0.00 \mathrm{~b}$ & $2.51 \pm 0.03 \mathrm{a}$ & $78.94 \pm 0.12 b$ \\
\hline & Temperature $\left({ }^{\circ} \mathrm{C}\right)$ & $p<0.01 *$ & $p=0.25 \mathrm{~ns}$ & $p=0.21 \mathrm{~ns}$ & $p<0.01 *$ & $p<0.01 *$ & $p<0.01 *$ & $p<0.01^{*}$ \\
\hline & 45 & $66.10 \pm 0.07 \mathrm{a}$ & $66.10 \pm 0.04 \mathrm{a}$ & $0.44 \pm 0.00 \mathrm{a}$ & $0.26 \pm 0.00 \mathrm{a}$ & $0.38 \pm 0.00 \mathrm{a}$ & $3.47 \pm 0.02 \mathrm{~b}$ & $71.50 \pm 0.10 \mathrm{a}$ \\
\hline & 80 & $83.51 \pm 0.07 \mathrm{~b}$ & $83.51 \pm 0.04 \mathrm{a}$ & $0.44 \pm 0.00 \mathrm{a}$ & $0.28 \pm 0.00 \mathrm{~b}$ & $0.48 \pm 0.00 \mathrm{~b}$ & $2.40 \pm 0.02 \mathrm{a}$ & $88.02 \pm 0.10 \mathrm{~b}$ \\
\hline & Mass (g) & $p<0.01 *$ & $p=0.89 \mathrm{~ns}$ & $p=0.53 \mathrm{~ns}$ & $p<0.01^{*}$ & $p<0.01 *$ & $p<0.01^{*}$ & $p<0.01 *$ \\
\hline & 1.5 & $73.19 \pm 0.07 a$ & $0.88 \pm 0.04 \mathrm{a}$ & $0.44 \pm 0.00 \mathrm{a}$ & $0.25 \pm 0.00 \mathrm{a}$ & $0.49 \pm 0.00 \mathrm{~b}$ & $3.59 \pm 0.02 b$ & $78.83 \pm 0.10 \mathrm{a}$ \\
\hline & 3.0 & $76.42 \pm 0.07 \mathrm{~b}$ & $0.87 \pm 0.04 \mathrm{a}$ & $0.44 \pm 0.00 \mathrm{a}$ & $0.30 \pm 0.00 \mathrm{~b}$ & $0.37 \pm 0.00 \mathrm{a}$ & $2.28 \pm 0.02 \mathrm{a}$ & $80.68 \pm 0.10 \mathrm{~b}$ \\
\hline \multirow{10}{*}{ UAE } & Time (min) & $p<0.01 *$ & $p=0.16 \mathrm{~ns}$ & $p<0.01 *$ & $p<0.01 *$ & $p<0.01 *$ & $p<0.01 *$ & $p<0.01 *$ \\
\hline & 7 & $76.90 \pm 0.17 \mathrm{~b}$ & $2.21 \pm 0.12 \mathrm{a}$ & $0.48 \pm 0.01 \mathrm{~b}$ & $0.41 \pm 0.02 \mathrm{~b}$ & $0.45 \pm 0.00 \mathrm{a}$ & $2.21 \pm 0.01 \mathrm{~b}$ & $82.67 \pm 0.21 \mathrm{~b}$ \\
\hline & 14 & $66.14 \pm 0.17 \mathrm{a}$ & $2.01 \pm 0.12 \mathrm{a}$ & $0.42 \pm 0.01 \mathrm{a}$ & $0.33 \pm 0.02 \mathrm{a}$ & $0.45 \pm 0.00 \mathrm{a}$ & $2.11 \pm 0.01 \mathrm{a}$ & $71.46 \pm 0.21 \mathrm{a}$ \\
\hline & 21 & $79.77 \pm 0.17 \mathrm{c}$ & $2.35 \pm 0.12 \mathrm{a}$ & $0.50 \pm 0.01 \mathrm{~b}$ & $0.44 \pm 0.02 \mathrm{~b}$ & $0.47 \pm 0.00 \mathrm{~b}$ & $2.27 \pm 0.01 \mathrm{c}$ & $85.79 \pm 0.21 \mathrm{c}$ \\
\hline & Amplitude (\%) & $p<0.01 *$ & $p=0.55 \mathrm{~ns}$ & $p=0.03 *$ & $p<0.01 *$ & $p<0.01 *$ & $p<0.01 *$ & $p<0.01 *$ \\
\hline & 50 & $72.18 \pm 0.14 \mathrm{a}$ & $2.23 \pm 0.09 a$ & $0.45 \pm 0.01 \mathrm{a}$ & $0.35 \pm 0.01 \mathrm{a}$ & $0.45 \pm 0.00 \mathrm{a}$ & $2.17 \pm 0.01 \mathrm{a}$ & $77.83 \pm 0.17 \mathrm{a}$ \\
\hline & 100 & $76.35 \pm 0.14 \mathrm{~b}$ & $2.15 \pm 0.09 a$ & $0.48 \pm 0.01 \mathrm{~b}$ & $0.43 \pm 0.01 \mathrm{~b}$ & $0.47 \pm 0.00 \mathrm{~b}$ & $2.22 \pm 0.01 \mathrm{~b}$ & $82.12 \pm 0.17 \mathrm{~b}$ \\
\hline & Mass (g) & $p<0.01 *$ & $p=0.02 *$ & $p<0.01 *$ & $p<0.01 *$ & $p<0.01 *$ & $p<0.01 *$ & $p<0.01 *$ \\
\hline & 1.5 & $72.79 \pm 0.14 \mathrm{a}$ & $2.37 \pm 0.09 \mathrm{~b}$ & $0.43 \pm 0.01 \mathrm{a}$ & $0.31 \pm 0.01 \mathrm{a}$ & $0.51 \pm 0.00 \mathrm{~b}$ & $2.35 \pm 0.01 \mathrm{~b}$ & $78.75 \pm 0.17 \mathrm{a}$ \\
\hline & 3.0 & $75.75 \pm 0.14 \mathrm{~b}$ & $2.02 \pm 0.09 \mathrm{a}$ & $0.51 \pm 0.01 \mathrm{~b}$ & $0.47 \pm 0.01 \mathrm{~b}$ & $0.40 \pm 0.00 \mathrm{a}$ & $2.05 \pm 0.01 \mathrm{a}$ & $81.20 \pm 0.17 b$ \\
\hline \multirow{11}{*}{ HPAE } & Time (min) & $p<0.01 *$ & $p=0.37 \mathrm{~ns}$ & $p=0.16 \mathrm{~ns}$ & $p=0.04$ * & $p<0.01 *$ & $p<0.01 *$ & $p<0.01 *$ \\
\hline & 1 & $68.91 \pm 0.29 \mathrm{~b}$ & $1.01 \pm 0.11 \mathrm{a}$ & $0.35 \pm 0.02 \mathrm{a}$ & $0.29 \pm 0.01 \mathrm{c}$ & $0.42 \pm 0.00 \mathrm{a}$ & $2.43 \pm 0.02 \mathrm{~b}$ & $73.41 \pm 0.31 \mathrm{~b}$ \\
\hline & 5.5 & $71.32 \pm 0.29 \mathrm{c}$ & $0.92 \pm 0.11 \mathrm{a}$ & $0.40 \pm 0.02 \mathrm{a}$ & $0.27 \pm 0.01 \mathrm{ab}$ & $0.44 \pm 0.00 \mathrm{~b}$ & $2.40 \pm 0.02 \mathrm{~b}$ & $75.76 \pm 0.31 \mathrm{c}$ \\
\hline & 10 & $67.46 \pm 0.29 a$ & $0.78 \pm 0.11 \mathrm{a}$ & $0.41 \pm 0.02 \mathrm{a}$ & $0.25 \pm 0.01 \mathrm{a}$ & $0.42 \pm 0.00 \mathrm{a}$ & $2.27 \pm 0.02 \mathrm{a}$ & $71.60 \pm 0.31 \mathrm{a}$ \\
\hline & Pressure (MPa) & $p<0.01$ * & $p=0.37 \mathrm{~ns}$ & $p=0.04^{*}$ & $p=0.19 \mathrm{~ns}$ & $p<0.01^{*}$ & $p=0.01$ * & $p<0.01^{*}$ \\
\hline & 300 & $68.06 \pm 0.23 \mathrm{a}$ & $0.96 \pm 0.09 \mathrm{a}$ & $0.42 \pm 0.02 \mathrm{~b}$ & $0.28 \pm 0.01 \mathrm{a}$ & $0.42 \pm 0.00 \mathrm{a}$ & $2.41 \pm 0.02 \mathrm{~b}$ & $72.54 \pm 0.25 \mathrm{a}$ \\
\hline & 500 & $70.40 \pm 0.23 \mathrm{~b}$ & $0.84 \pm 0.09 \mathrm{a}$ & $0.36 \pm 0.02 \mathrm{a}$ & $0.26 \pm 0.01 \mathrm{a}$ & $0.44 \pm 0.00 \mathrm{~b}$ & $2.33 \pm 0.02 \mathrm{a}$ & $74.64 \pm 0.25 b$ \\
\hline & Mass (g) & $p<0.01^{*}$ & $p=0.34 \mathrm{~ns}$ & $p=0.01^{*}$ & $p<0.01^{*}$ & $p<0.01^{*}$ & $p<0.01^{*}$ & $p<0.01 *$ \\
\hline & 1.5 & $71.28 \pm 0.23 b$ & $0.97 \pm 0.09 \mathrm{a}$ & $0.43 \pm 0.02 \mathrm{~b}$ & $0.25 \pm 0.01 \mathrm{a}$ & $0.50 \pm 0.00 \mathrm{~b}$ & $2.59 \pm 0.02 \mathrm{~b}$ & $76.02 \pm 0.25 b$ \\
\hline & 3.0 & $67.18 \pm 0.23 a$ & $0.84 \pm 0.09 \mathrm{a}$ & $0.35 \pm 0.02 \mathrm{a}$ & $0.30 \pm 0.01 \mathrm{~b}$ & $0.36 \pm 0.00 \mathrm{a}$ & $2.15 \pm 0.02 \mathrm{a}$ & $71.16 \pm 0.25 \mathrm{a}$ \\
\hline & 300 & $68.06 \pm 0.23 a$ & $0.96 \pm 0.09 a$ & $0.42 \pm 0.02 \mathrm{~b}$ & $0.28 \pm 0.01 \mathrm{a}$ & $0.42 \pm 0.00 \mathrm{a}$ & $2.41 \pm 0.02 \mathrm{~b}$ & $72.54 \pm 0.25 \mathrm{a}$ \\
\hline
\end{tabular}

$\mathrm{MAE}=$ microwave-assisted extraction, $\mathrm{UAE}=$ ultrasound-assisted extraction, $\mathrm{HPAE}=$ high pressure-assisted extraction, $\mathrm{CE}=$ conventional heat-reflux extraction, $\mathrm{OL}=$ oleuropein,
$\mathrm{HT}=$ hydroxytyrosol, $\mathrm{CA}=$ chlorogenic acid $\mathrm{CF}=$ caffeic acid $\mathrm{VB}=$ verbascoside, $\mathrm{RT}=$ rutin ${ }^{*} p \leq 0.05, \mathrm{~ns}=$ not significant $(p>0.05)$. Results are expressed as mean $\pm \mathrm{SE}$. Values with different letters within column are statistically different at $p \leq 0.05$. 
Sample mass also had a significant $(p \leq 0.05)$ influence on all identified polyphenols, except HT and CA. According to Spigno and De Faveri (2009) [16], if the solvent volume is maintained constant (changing only solids content), the temperature of the MAE system is constant which can result in a higher extraction yield at lower solid/solvent ratios. Indeed, higher VB and RT concentrations were achieved at lower solid/solvent ratios (1.5 g of sample). However, OL and CF concentrations were higher when using $3 \mathrm{~g}$ of the sample, which can be considered as a positive environmental effect since the consumption of solvent is lower.

\subsection{Ultrasound-Assisted Extraction (UAE)}

Extraction time (7, 14, and $21 \mathrm{~min})$, amplitude (50 and 100\%), as well as sample mass (1.5 and $3 \mathrm{~g}$ ) were varied during UAE of olive leaves polyphenols. Obtained results were statistically analyzed and are given in Table 2. Time had a significant $(p \leq 0.05)$ influence on all polyphenols except HT and CF. The highest total polyphenols, OL, VB, and RT concentrations were obtained after $21 \mathrm{~min}$, while the lowest OL, RT, and CA concentrations were obtained after $14 \mathrm{~min}$. By applying a longer extraction time, the sample is in longer contact with the solvent, which facilitates a higher diffusion of the target compounds [11]. However, the overexposure to the ultrasound treatment can cause the heating effect, which leads to the degradation of some polyphenols [29]. Some authors [30,31] reported a positive effect of UAE time on the extraction yield in the blackthorn flower (Prunus spinosa L.) and sage (Salvia officinalis L.) extracts, while others reported that longer UAE time led to the lower extraction yield in banana peel [32]. Composition and stability of polyphenols vary in different types of material, which also explains these differences in the reported results. The temperature difference between MAE $\left(80^{\circ} \mathrm{C}\right)$ and UAE $\left(60^{\circ} \mathrm{C}\right)$ could explain why RT showed high sensitivity to elevated temperatures during longer extraction times in MAE, while the highest RT concentration in UAE was obtained even after $21 \mathrm{~min}$ (Table 2). In general, the optimal extraction time for the majority of OLE polyphenols was much shorter in MAE (2 min) than UAE (21 min), similarly to Hannachi et al. (2019) [14] who noted that optimal MAE and UAE time for olive leaves polyphenols was 1.81 and $28.69 \mathrm{~min}$, respectively.

Amplitude also had an important role in the extraction of all identified polyphenols except HT and CF. Using 100\% amplitude resulted in higher concentrations of all polyphenols influenced by this parameter, since more cell walls are damaged by applying higher ultrasound amplitude, therefore, causing the release of more polyphenols to the solvent [11].

Considering the sample mass, all identified polyphenols were significantly affected $(p \leq 0.05)$ by the amount of sample. Higher OL, CF, and CA concentrations were achieved by using $3 \mathrm{~g}$ of the sample, while higher RT, VB, and HT concentrations were achieved with lower sample mass $(1.5 \mathrm{~g})$. This difference could be linked with the specificity of an individual compound, although the increase of RT, VB, and HT yields with the solvent/sample ratio increase is consistent with the mass transfer principles, where the concentration gradient, which is the driving force, is higher when a lower solid/solvent ratio is used, thus leading to higher diffusion [1]. Sample mass in UAE had the same effect on OLE polyphenols as in MAE, where $3 \mathrm{~g}$ of the sample yielded the highest total sum of all polyphenols indicating a lower solvent consumption. Contrarily, Şahin and Şamlı (2013) [1] achieved higher olive leaves phenolic content in UAE extract by using a lower solid/solvent ratio.

\subsection{High Pressure-Assisted Extraction (HPAE)}

Table 2 provides the results of HPAE time (1,5.5, and $10 \mathrm{~min}$ ), pressure (300 and $500 \mathrm{MPa}$ ) and sample mass (1.5 and $3 \mathrm{~g}$ ) effect on the OLE polyphenols. Pressure holding time had a significant $(p \leq 0.05)$ influence on all polyphenols, except HT and CA. Its main function is to form the equilibrium of solvent concentration between the inner and outer part of the cells and to get in full contact with the bioactive compounds and solvent [12]. Pressure holding time showed a similar influence on OL and VB concentration-with the time increase, from 1 to $5.5 \mathrm{~min}$, concentrations of these compounds increased, but with a further increase $(10 \mathrm{~min})$ they decreased. Similar findings were previously reported by Chen et al. (2009) [33] in HPAE of ginsenosides from roots of ginseng. By increasing the extraction 
time from 1 to $5 \mathrm{~min}$, the yield of extracted saponins increased, but when extractions were longer than 5 min the yield was kept constant. According to Pascal's theory [12], during the high hydrostatic pressure (HHP) treatment, the pressure uniformly and instantly transfers to the whole material, which makes the extraction process fast, easy and effective. In comparison with MAE, where the optimal extraction time for total polyphenols was $2 \mathrm{~min}$, HPAE optimal extraction time was slightly higher (5.5 $\mathrm{min}$ ), whereas it was much shorter than the $21 \mathrm{~min}$ required for optimal UAE (Table 2). However, total polyphenols content extracted by HPAE in the optimal time was much lower than those obtained in the optimal MAE and UAE time (Table 2).

Furthermore, from Table 2 it can be observed that OL and VB concentrations were significantly higher at $500 \mathrm{MPa}$, while RT and CA concentrations were significantly higher at $300 \mathrm{MPa}$. Diverse data on optimal pressure were also previously reported [33-35] and Corrales et al. (2009) [36] described that due to the similar degree of cell membrane disruption, the content of anthocyanins extracted from grape skins did not significantly differ $(p>0.05)$ upon different pressure intensities. HHP can enhance polyphenols' extraction by causing several different effects, where it will disrupt the cell wall and release the cytoplasm, which contains a high concentration of target material [12]. By applying HHP more solvent can permeate into the cells and as a consequence, more compounds can diffuse into the solvent [12]. Furthermore, the solubility of extracts is improved as the pressure increases [12]. A common trend that can be observed between MAE, UAE, and HPAE is that when a parameter that enhances a cell wall disruption (temperature in MAE (influenced by microwave power); amplitude in UAE; pressure in HPAE) is elevated, the total sum of the extracted polyphenols is higher (Table 2).

Sample mass showed to have an important role in the extraction of all identified compounds except HT (Table 2). It can be observed that OL, RT, VB, and CA concentrations were significantly higher when using $1.5 \mathrm{~g}$ of the sample, where an evidently lower sample mass/solvent ratio enhanced the possibility for bioactive compounds to get into contact with the extraction solvent. Since the dissolving process of bioactive compounds into the solvent is a physical process, it leads to higher leaching-out rates $[12,35]$. In general, using a higher ratio of solvent when compared to raw material gives a more dilute effect on the solvent side. This gives a larger concentration difference between the interior of the plant cells and the external solvent and, therefore, a faster extraction rate can be achieved [33]. While $1.5 \mathrm{~g}$ of the sample was the optimal sample mass in HPAE, the opposite result was obtained in MAE and UAE where a mass of $3 \mathrm{~g}$ resulted with higher total polyphenols extraction (Table 2).

In summary, based on the statistical analysis, results showed that optimal values of parameters that promote the highest total polyphenols extraction efficiency were $2 \mathrm{~min} / 80{ }^{\circ} \mathrm{C} / 3 \mathrm{~g}$ in $\mathrm{MAE}$, $21 \mathrm{~min} / 100 \% / 3 \mathrm{~g}$ in UAE, and $5.5 \mathrm{~min} / 500 \mathrm{MPa} / 1.5 \mathrm{~g}$ in HPAE.

\subsection{Mutual Comparison of Advanced Extraction Techniques and with Conventional Heat-Reflux}

In order to provide a better insight into the efficiency of tested extraction techniques, all techniques were mutually compared, as well as with the conventional heat-reflux. The comparison of grand mean values obtained for olive leaves individual polyphenols and their sum isolated by various advanced extraction techniques and the mean values of analyzed compounds and their sum for heat-reflux is presented in Table 3. When compared mutually, it can be seen that almost all polyphenols' yields were the highest when extracted by MAE and UAE, especially OL as the most abundant, while the ones obtained by HPAE were slightly lower. Moreover, the comparison of polyphenols' total sum clearly shows that the highest levels of polyphenols were achieved in MAE and UAE extracts (Table 3).

When compared to the CE, the total sum of all identified polyphenols, as well as OL and RT concentrations were the lowest when CE was used (Table 3). Similarly, Hannachi et al. (2019) [14] noted that when compared to the CE methods, MAE and UAE were more efficient for the extraction of polyphenols from olive leaves. What is often described as a highlight of advanced techniques is the lower level of biomaterial's heating in comparison with the CE. Since many organic compounds, 
including polyphenols, are heat-sensitive, by applying a high temperature-extraction technique for a longer time they will be denatured, lose biologic activity, or change into another compound [37].

Table 3. Comparison of olive leaves individual phenols $\left(\mathrm{mg} \mathrm{g}^{-1}\right)$ extracted with various extraction techniques.

\begin{tabular}{cccccccc}
\hline $\begin{array}{c}\text { Extraction } \\
\text { Technique }\end{array}$ & OL & HT & CA & CF & VB & RT & Total Sum \\
\hline MAE & $74.81 \pm 2.12$ & $0.87 \pm 0.03$ & $0.44 \pm 0.00$ & $0.27 \pm 0.01$ & $0.43 \pm 0.02$ & $2.94 \pm 0.36$ & $79.76 \pm 1.92$ \\
UAE & $74.27 \pm 1.90$ & $2.19 \pm 0.10$ & $0.47 \pm 0.02$ & $0.39 \pm 0.03$ & $0.46 \pm 0.01$ & $2.20 \pm 0.05$ & $79.97 \pm 2.01$ \\
HPAE & $69.23 \pm 0.87$ & $0.90 \pm 0.07$ & $0.39 \pm 0.02$ & $0.27 \pm 0.01$ & $0.43 \pm 0.02$ & $2.37 \pm 0.05$ & $73.59 \pm 0.93$ \\
CE & $67.05 \pm 1.21$ & $0.89 \pm 0.07$ & $0.41 \pm 0.01$ & $0.27 \pm 0.01$ & $0.47 \pm 0.02$ & $2.01 \pm 0.06$ & $71.11 \pm 1.30$ \\
\hline
\end{tabular}

$\mathrm{MAE}=$ microwave-assisted extraction, $\mathrm{UAE}=$ ultrasound-assisted extraction, HPAE $=$ high pressure-assisted extraction, $\mathrm{CE}=$ conventional heat-reflux extraction, $\mathrm{OL}=$ oleuropein, $\mathrm{HT}=$ hydroxytyrosol, $\mathrm{CA}=$ chlorogenic acid, $\mathrm{CF}=$ caffeic acid, $\mathrm{VB}=$ verbascoside, $\mathrm{RT}=$ rutin. For MAE, $\mathrm{UAE}$, and HPAE results are expressed as mean $\pm \mathrm{SE}$, for $\mathrm{CE}$ results are expressed as mean $\pm \mathrm{SD}$.

In addition to the lowest total polyphenols' yield obtained by $\mathrm{CE}$ (Table 3), the crucial difference between $\mathrm{CE}$ and all other advanced extraction techniques applied in this study was a significant reduction of time. A time of $60 \mathrm{~min}$ in CE (two cycles of $30 \mathrm{~min}$ ), as its optimal extraction condition, was required to extract the maximum yield of polyphenols, which was lower when compared to the concentrations of polyphenols extracted in the optimal time of 2, 21, or $5.5 \mathrm{~min}$ in MAE, UAE, and HPAE (Table 2), respectively.

Comparing the results in Table 3, much higher concentrations of all identified compounds were achieved with MAE (79.76 $\left.\mathrm{mg} \mathrm{g}^{-1}\right)$ and UAE $\left(79.97 \mathrm{mg} \mathrm{g}^{-1}\right)$ than with HPAE $\left(73.59 \mathrm{mg} \mathrm{g}^{-1}\right)$ and CE $\left(71.11 \mathrm{mg} \mathrm{g}^{-1}\right)$. Therefore, similar polyphenols' extraction was achieved at a lower temperature $\left(60^{\circ} \mathrm{C}\right)$ applied for a longer time $(21 \mathrm{~min})$ in UAE and higher temperature $\left(80^{\circ} \mathrm{C}\right)$ applied for a shorter time (2 min) in MAE. In research by Hannachi et al. (2019) [14], a higher olive leaves total polyphenols' content was achieved with MAE when compared to UAE.

Regarding the effect of different extraction processes on individual polyphenols, an interesting observation was that HT concentration was not influenced by any of the applied extraction parameters. The reason could be that HT is an OL and VB moiety, and can be obtained by their degradation. OL degradation occurs by the opening of the oleanolic acid ring with a final rearrangement into HT [38]. Further, results of Briante et al. (2000) [39] indicate high stability of HT, since it was not qualitatively degraded when heated at $70{ }^{\circ} \mathrm{C}$ for $3 \mathrm{~h}$, while OL aglycon disappeared after $30 \mathrm{~min}$ at $70{ }^{\circ} \mathrm{C}$. Contrarily, RT seems to be the least stable compound throughout all of the examined extraction techniques. Furthermore, HT concentration was more than two-fold higher by UAE when compared to the other extraction techniques. Likewise, Xie et al. (2019) [40] considered UAE the most effective method for HT extraction from olive pomace.

Additionally, the possible grouping of OLE samples regarding the applied extraction technique was tested using PCA. Obtained results are presented in Figure 3, which provides the PCA biplot based on the MANOVA results (Table 2) giving a better visual perception of the extracts grouping by the applied extraction techniques. The first two components (PC1 and PC2) explained $65.30 \%$ of the total variance. As it can be seen, PCA showed a certain grouping of OLE, where most of the samples obtained using MAE and UAE were situated at negative values of PC1, while HPAE and CE samples as well, were situated at PC1 positive values of the biplot. All identified polyphenols, except RT, were considered as the most discriminating variables due to the strong/very strong correlation $(\mathrm{r}=0.65-0.90)$ with both principal components, which is in accordance with previously discussed data (Tables 1-3). 


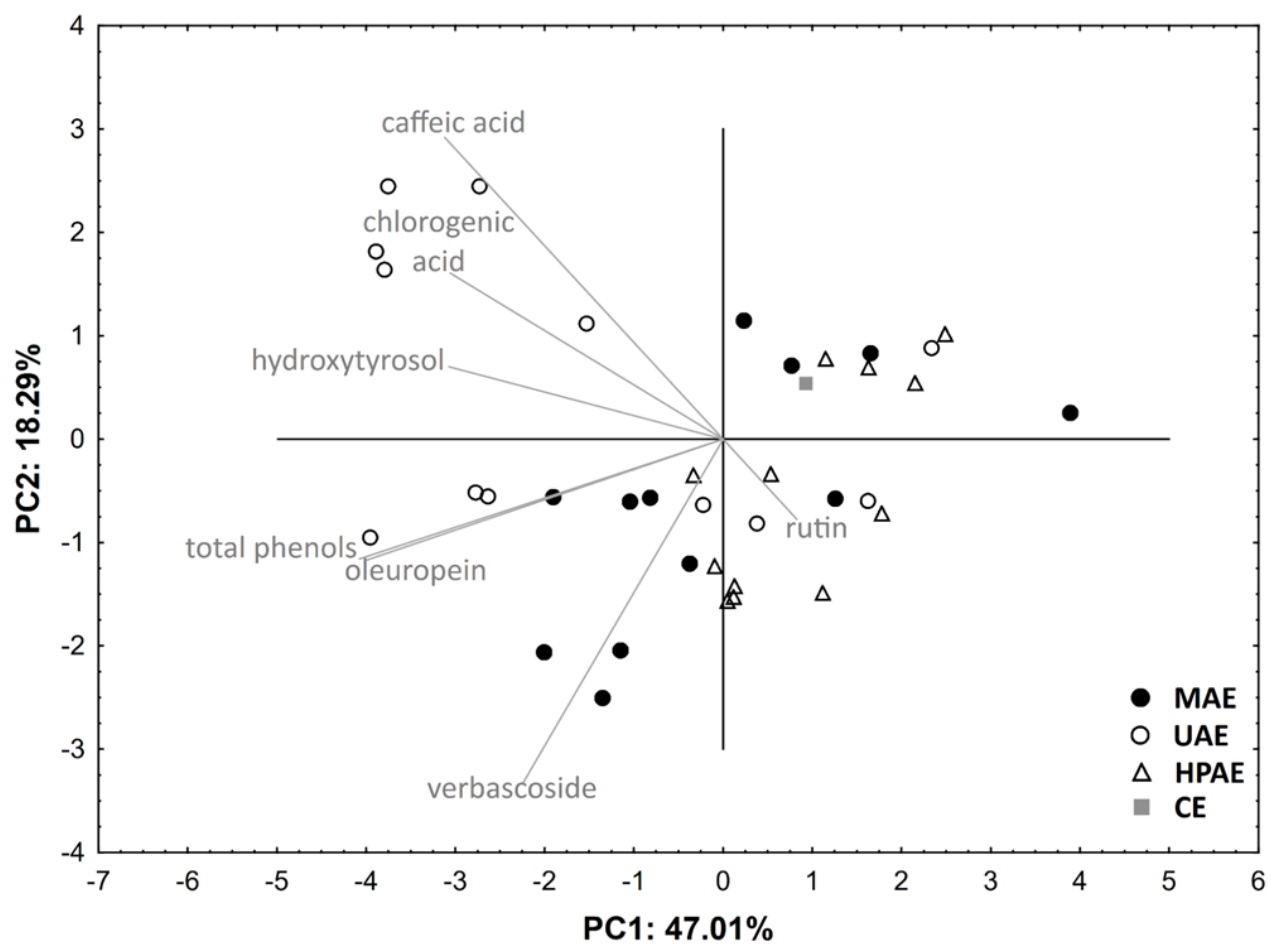

Figure 3. Distribution of olive leaves extracts in a two-dimensional coordinate system defined by the first two principal components (PC1 and PC2) according to the applied extraction technique $(\mathrm{MAE}=$ microwave-assisted extraction, $\mathrm{UAE}=$ ultrasound-assisted extraction, $\mathrm{HPAE}=$ high pressure-assisted extraction, $\mathrm{CE}=$ conventional heat-reflux extraction).

\section{Conclusions}

Olive leaves extracts obtained using MAE, UAE, and HPAE consisted of HT, CA, CF, VB, RT and OL. The most abundant polyphenol in all extracts was OL (83.59-95.20\%) with an average concentration of 74.81 (MAE), 74.27 (UAE), and $69.23 \mathrm{mg} \mathrm{g}^{-1}$ (HPAE). Except for HT which was not influenced by any of the extraction parameters and RT which showed high sensitivity, statistical analysis showed that optimal values of parameters that resulted with the highest efficiency of olive leaves polyphenols' extraction were $2 \mathrm{~min} / 80^{\circ} \mathrm{C} / 3 \mathrm{~g}$ in MAE, $21 \mathrm{~min} / 100 \% / 3 \mathrm{~g}$ in UAE, and $5.5 \mathrm{~min} / 500 \mathrm{MPa} / 1.5 \mathrm{~g}$ in HPAE. Considering the average total polyphenols' content, MAE $\left(79.76 \mathrm{mg} \mathrm{g}^{-1}\right)$ and UAE $\left(79.97 \mathrm{mg} \mathrm{g}^{-1}\right)$ were more efficient in comparison with HPAE $\left(73.59 \mathrm{mg} \mathrm{g}^{-1}\right)$. Furthermore, all three alternative extraction techniques yielded a higher content of total polyphenols when compared to the CE $\left(71.11 \mathrm{mg} \mathrm{g}^{-1}\right)$ along with a significant reduction in time from $60 \mathrm{~min}$ in CE to 2, 21, and $5.5 \mathrm{~min}$ in MAE, UAE, and HPAE, respectively. Combining all obtained results, MAE and UAE could have a slight advantage over HPAE and could be successfully used for the recovery of olive leaves polyphenols with MAE being less time-consuming.

Author Contributions: Conceptualization, A.D., L.T. and B.L.; Data curation, A.D., M.R. and L.T.; Formal analysis, A.D., M.R. and L.T.; Investigation, A.D., L.T. and B.L.; Methodology, A.D., L.T. and B.L.; Project administration, V.D.-U.; Supervision, B.L.; Writing-original draft, A.D., M.R. and I.E.G.; Writing—review and editing, V.D.-U. and B.L. All authors have read and agreed to the published version of the manuscript.

Funding: This work was supported by the project "Bioactive molecules of medical plants as natural antioxidants, microbicides, and preservatives" (KK.01.1.1.04.0093), co-financed by the Croatian Government and the European Union through the European Regional Development Fund-Operational Programme Competitiveness and Cohesion (KK.01.1.1.04.).

Conflicts of Interest: The authors declare no conflict of interest. The funders had no role in the design of the study; in the collection, analyses, or interpretation of data; in the writing of the manuscript, or in the decision to publish the results. 


\section{References}

1. Sahin, S.; Şamli, R. Optimization of olive leaf extract obtained by ultrasound-assisted extraction with response surface methodology. Ultrason. Sonochem. 2013, 20, 595-602. [CrossRef] [PubMed]

2. Ben, S.M.; Abdelmelek, H. Study of phenolic composition and biological activities assessment of olive leaves from different varieties grown in Tunisia. Med. Chem. 2012, 2, 107-111. [CrossRef]

3. Talhaoui, N.; Gómez-Caravaca, A.M.; León, L.; De la Rosa, R.; Segura-Carretero, A.; Fernández-Gutiérrez, A. Determination of phenolic compounds of "Sikitita" olive leaves by HPLC-DAD-TOF-MS. Comparison with its parents "Arbequina" and "Picual" olive leaves. LWT Food Sci. Technol. 2014, 58, 28-34. [CrossRef]

4. Taamalli, A.; Arráez-Román, D.; Ibañez, E.; Zarrouk, M.; Segura-Carretero, A.; Fernández-Gutiérrez, A. Optimization of microwave-assisted extraction for the characterization of olive leaf phenolic compounds by using HPLC-ESI-TOF-MS/IT-MS2. J. Agric. Food Chem. 2012, 60, 791-798. [CrossRef] [PubMed]

5. Mkaouar, S.; Gelicus, A.; Bahloul, N.; Allaf, K.; Kechaou, N. Kinetic study of polyphenols extraction from olive (Olea europaea L.) leaves using instant controlled pressure drop texturing. Sep. Purif. Technol. 2016, 161, 165-171. [CrossRef]

6. Quirantes-Piné, R.; Lozano-Sánchez, J.; Herrero, M.; Ibáñez, E.; Segura-Carretero, A.; Fernández-Gutiérrez, A. HPLC-ESI-QTOF-MS as a powerful analytical tool for characterising phenolic compounds in olive-leaf extracts. Phytochem. Anal. 2013, 24, 213-223. [CrossRef]

7. Bouaziz, M.; Sayadi, S. Isolation and evaluation of antioxidants from leaves of a Tunisian cultivar olive tree. Eur. J. Lipid Sci. Technol. 2005, 107, 497-504. [CrossRef]

8. Mitsopoulos, G.; Hagidimitriou, M.; Papageorgiou, V.; Komaitis, M. Total phenolic content, phenolic profile and antioxidant activity in leaves and drupes of Greek olive cultivars. Acta Hortic. 2011, 924, 425-430. [CrossRef]

9. Huang, H.W.; Hsu, C.P.; Yang, B.B.; Wang, C.Y. Advances in the extraction of natural ingredients by high pressure extraction technology. Trends Food Sci. Technol. 2013, 33, 54-62. [CrossRef]

10. Veggi, P.C.; Martinez, J.; Meireles, M.A.A. Fundamentals of Microwave Extraction. In Microwave-Assisted Extraction for Bioactive Compounds: Theory and Practice; Chemat, F., Cravotto, G., Eds.; Springer Science + Business Media: New York, NY, USA, 2013; ISBN 978-1-4614-4829-7.

11. Hossain, M.; Brunton, N.; Patras, A.; Tiwari, B.; O’Donnell, C.; Martin-Diana, A.B.; Barry-Ryan, C. Optimization of ultrasound assisted extraction of antioxidant compounds from marjoram (Origanum majorana L.) using response surface methodology. Ultrason. Sonochem. 2012, 19, 582-590. [CrossRef]

12. Xi, J.; Shen, D.; Zhao, S.; Lu, B.; Li, Y.; Zhang, R. Characterization of polyphenols from green tea leaves using a high hydrostatic pressure extraction. Int. J. Pharm. 2009, 382, 139-143. [CrossRef] [PubMed]

13. Ahmad-Qasem, M.H.; Cánovas, J.; Barrajón-Catalán, E.; Micol, V.; Cárcel, J.A.; García-Pérez, J.V. Kinetic and compositional study of phenolic extraction from olive leaves (var. Serrana) by using power ultrasound. Innov. Food Sci. Emerg. Technol. 2013, 17, 120-129. [CrossRef]

14. Hannachi, H.; Benmoussa, H.; Saadaoui, E.; Saanoun, I.; Negri, N.; Elfalleh, W. Optimization of ultrasound and microwave-assisted extraction of phenolic compounds from olive leaves by response surface methodology. Res. J. Biotechnol. 2019, 14, 28-37.

15. Rafiee, Z.; Jafari, S.M.; Alami, M.; Khomeiri, M. Microwave-Assisted extraction of phenolic compounds from olive leaves, A comparison with maceration. J. Anim. Plant Sci. 2011, 21, 738-745.

16. Spigno, G.; De Faveri, D.M. Microwave-Assisted extraction of tea phenols: A phenomenological study. J. Food Eng. 2009, 93, 210-217. [CrossRef]

17. Buzrul, S.; Alpas, H.; Largeteau, A.; Bozoglu, F.; Demazeau, G. Compression heating of selected pressure transmitting fluids and liquid foods during high hydrostatic pressure treatment. J. Food Eng. 2008, 85, 466-472. [CrossRef]

18. Richard, N.; Arnold, S.; Hoeller, U.; Kilpert, C.; Wertz, K.; Schwager, J. Hydroxytyrosol is the major anti-inflammatory compound in aqueous olive extracts and impairs cytokine and chemokine production in macrophages. Planta Med. 2011, 77, 1890-1897. [CrossRef]

19. Damtoft, S.; Franzyk, H.; Jensen, S.R. Biosynthesis of secoiridoid glucosides in Oleaceae. Phytochemistry 1993, 34, 1291-1299. [CrossRef]

20. Visioli, F.; Galli, C. The effect of minor constituents of olive oil on cardiovascular disease: New findings. Nutr. Rev. 1998, 56, 142-147. [CrossRef] 
21. Le Tutour, B.; Guedon, D. Antioxidative activities of Olea europaea leaves and related phenolic compounds. Phytochemistry 1992, 31, 1173-1178. [CrossRef]

22. Benavente-Garcia, O.; Castillo, J.; Lorente, J.; Ortuño, A.D.R.J.; Del Rio, J.A. Antioxidant activity of phenolics extracted from Olea europaea L. leaves. Food Chem. 2000, 68, 457-462. [CrossRef]

23. Dekanski, D.; Janicijevic-Hudomal, S.; Tadic, V.; Markovic, G.; Arsic, I.; Mitrovic, D. Phytochemical analysis and gastroprotective activity of an olive leaf extract. J. Serb. Chem. Soc. 2009, 74, 367-377. [CrossRef]

24. Japón-Luján, R.; Luque-Rodríguez, J.M.; De Castro, M.D.L. Multivariate optimisation of the microwave-assisted extraction of oleuropein and related biophenols from olive leaves. Anal. Bioanal. Chem. 2006, 385, 753-759. [CrossRef] [PubMed]

25. Mandal, V.; Mohan, Y.; Hemalatha, S. Microwave assisted extraction-An innovative and promising extraction tool for medicinal plant research. Pharmacogn. Rev. 2007, 1, 7-18.

26. Murakami, M.; Yamaguchi, T.; Takamura, H.; Atoba, T.M. Effects of thermal treatment on radical-scavenging activity of single and mixed polyphenolic compounds. J. Food Sci. 2004, 69, FCT7-FCT10. [CrossRef]

27. Ioannou, I.; Ghoul, M. Biological activities and effects of food processing on flavonoids as phenolics antioxidants. Adv. Appl. Biotechnol. 2012, 101-124. [CrossRef]

28. Pacheco-Palencia, L.A.; Mertens-Talcott, S.; Talcott, S.T. Chemical composition, antioxidant properties, and thermal stability of a phytochemical enriched oil from Açai (Euterpe oleracea Mart.). J. Agric. Food Chem. 2008, 56, 4631-4636. [CrossRef]

29. Chimsook, T.; Wannalangka, W. Effect of ultrasonic-assisted extraction on phenolic content of freshwater macroalgae in northern Thailand. In Proceedings of the MATEC Web of Conferences, Lisbon, Portugal, 23-25 November 2015; Volume 35, p. 04002. [CrossRef]

30. Elez, G.I.; Zorić, Z.; Pedisić, S.; Brnčić, M.; Dragović-Uzelac, V. UPLC-MS2 profiling of blackthorn flower polyphenols isolated by ultrasound-assisted extraction. J. Food Sci. 2018, 83, 2782-2789. [CrossRef]

31. Dent, M.; Dragovic-Uzelac, V.; Garofulic, I.E.; Bosiljkov, T.; Ježek, D.; Brncic, M. Comparison of conventional and ultrasound-assisted extraction techniques on mass fraction of phenolic compounds from sage (Salvia officinalis L.). Chem. Biochem. Eng. Q. 2015, 29, 475-484. [CrossRef]

32. Vu, H.T.; Scarlett, C.J.; Vuong, Q.V. Optimization of ultrasound-assisted extraction conditions for recovery of phenolic compounds and antioxidant capacity from banana (Musa cavendish) peel. J. Food Process. Preserv. 2016, 2016, e13148. [CrossRef]

33. Chen, R.; Meng, F.; Zhang, S.; Liu, Z. Effects of ultrahigh pressure extraction conditions on yields and antioxidant activity of ginsenoside from ginseng. Sep. Purif. Technol. 2009, 66, 340-346. [CrossRef]

34. Butz, P.; Koller, W.D.; Tauscher, B.; Wolf, S. Ultra-High pressure processing of onions: Chemical and sensory changes. LWT Food Sci. Technol. 1994, 27, 463-467. [CrossRef]

35. Prasad, K.N.; Yang, B.; Zhao, M.; Wei, X.; Jiang, Y.; Chen, F. High pressure extraction of corilagin from longan (Dimocarpus longan Lour.) fruit pericarp. Sep. Purif. Technol. 2009, 70, 41-45. [CrossRef]

36. Corrales, M.; García, A.F.; Butz, P.; Tauscher, B. Extraction of anthocyanins from grape skins assisted by high hydrostatic pressure. J. Food Eng. 2009, 90, 415-421. [CrossRef]

37. Shouqin, Z.; Junjie, Z.; Changzhen, W. Novel high pressure extraction technology. Int. J. Pharm. 2004, 278, 471-474. [CrossRef] [PubMed]

38. Afaneh, I.; Yateem, H.; Al-Rimawi, F. Effect of Olive Leaves Drying on the Content of Oleuropein. Am. J. Anal. Chem. 2015, 6, 246-252. [CrossRef]

39. Briante, R.; La Cara, F.; Febbraio, F.; Barone, R.; Piccialli, G.; Carolla, R.; Mainolfi, P.; De Napoli, L.; Patumi, M.; Fontanazza, G.; et al. Hydrolysis of oleuropein by recombinant b-glycosidase from hyperthermophilic archaeon. J. Biotechnol. 2000, 77, 275-286. [CrossRef]

40. Xie, P.; Huang, L.; Zhang, C.; Deng, Y.; Wang, X.; Cheng, J. Enhanced extraction of hydroxytyrosol, maslinic acid and oleanolic acid from olive pomace: Process parameters, kinetics and thermodynamics, and greenness assessment. Food Chem. 2019, 276, 662-674. [CrossRef]

(C) 2020 by the authors. Licensee MDPI, Basel, Switzerland. This article is an open access article distributed under the terms and conditions of the Creative Commons Attribution (CC BY) license (http://creativecommons.org/licenses/by/4.0/). 